\title{
GMR
}

\section{Activation of p38-mitogen-activated protein kinase contributes to ischemia reperfusion in rat brain}

\author{
Y.Q. Song', H.L. Zou' ${ }^{2}$, Y.J. Zhao ${ }^{1}$, L.Q. Yu ${ }^{1}$, Z.X. $\operatorname{Tan}^{1}$ and R. Kong ${ }^{1}$ \\ ${ }^{1}$ Department of Neurology, The Affiliated Hospital of Qingdao University, \\ Qingdao, Shandong, China \\ ${ }^{2}$ Department of Internal Medicine, Qingdao Central Hospital, Qingdao, \\ Shandong, China \\ Corresponding author: H.L. Zou \\ E-mail: honglizoucn@126.com
}

Genet. Mol. Res. 15 (3): gmr.15038492

Received January 26, 2016

Accepted April 8, 2016

Published September 23, 2016

DOI http://dx.doi.org/10.4238/gmr.15038492

Copyright (C) 2016 The Authors. This is an open-access article distributed under the terms of the Creative Commons Attribution ShareAlike (CC BY-SA) 4.0 License.

\begin{abstract}
Inflammation plays an important role in cerebral ischemia reperfusion, which can cause severe damage to the brain and may lead to cerebral hemorrhage transformation. p38-mitogen-activated protein kinase (p38mapk) has been implicated in the etiology of a number of diseases because it is a cause of inflammation, but comparatively little research has been carried out into its role in the etiology of ischemia reperfusion. We investigated the expression of p38mapk in cerebral ischemia reperfusion to gain a better understanding of its potential role in hemorrhagic transformation (HT). One hundred rats were randomly divided into three groups: an ischemia reperfusion group, an ischemia group, and a sham-operated group. We carried out neurological deficit assessments, infarct volume measurements, histopathological examinations, and immunohistochemistry analyses. p38mapk was
\end{abstract}


overexpressed in the ischemia reperfusion group, which exhibited severe tissue damage and greater edema than the other two groups. These results suggest that $\mathrm{p} 38 \mathrm{mapk}$ plays an important role in cerebral ischemia reperfusion, and may be one of the causes of HT.

Key words: Cerebral ischemia; Inflammation; p38mapk

\section{INTRODUCTION}

Cerebral infarction is a serious threat to human life, especially in adults. In patients with cerebral ischemia, recanalization is the most effective means of treating acute cerebral infarct. Emergency medical interventions for cerebral infarction include intravenous thrombolysis, endovascular interventions, systemic anticoagulation, and antiplatelet therapy (Robinson et al., 2014). The therapeutic goal is to salvage potentially reversible ischemic tissue and save the ischemic penumbra in order to reduce infarct size and improve neuromuscular function. However, recanalization carries a risk of hemorrhagic transformation (HT).

Recent studies have shown that disruption of the blood brain barrier (BBB) leads to HT. Therefore, preservation of the BBB is a common goal of neuroprotective therapies (Wang et al., 2014a). HT may be caused by cerebral ischemia reperfusion, which is a complex pathological condition in which the inflammatory response plays a crucial role (Wang et al., 2013). Cerebral ischemia reperfusion and local cell debris have a strong cytotoxic effect in the brain parenchyma after middle cerebral artery occlusion (MCAO). The cytotoxic response occurs within minutes of the onset of cerebral ischemia and involves proinflammatory responses, oxidative stress, neurologic damage, and cell death (Choi et al., 2010). Inflammation leads to increased permeability of the BBB, and blood may be discharged from damaged vessels. Therefore, inhibition of inflammatory responses to protect the BBB at the early stage of ischemia is an attractive therapeutic strategy.

Mitogen-activated protein kinases (MAPKs) are serine/threonine kinases that are rapidly activated in response to growth factor stimulation. The MAPK pathway is an important intracellular signal transduction system that involves extracellular signal-regulated protein kinase (ERK), stress-activated protein kinases, or c-jun, N-terminal kinase (JNK), and p38mitogen-activated protein kinase (p38mapk) (Exil et al., 2014; Wang et al., 2014b). p38mapk is involved in intracellular responses, such as inflammation, cell-cycle regulation, cell death, development, differentiation, senescence, and tumorigenesis (Zhao et al., 2014). In one study using a mouse model of allergic asthma, treatment with p38mapk and inducible nitric oxide synthase inhibitors attenuated the effects of glutathione depletion on airway reactivity and inflammation (Nadeem et al., 2014).

There are few therapeutic strategies available for minimizing brain damage and improving functional recovery after a stroke. Similarly, few therapeutic strategies are available for HT. Neural therapy is used to treat cerebral hemorrhage, but it is a negative therapy. Cerebral hemorrhage is normally treated using neutral methods, but no significant effect has ever been found.

There is a need to develop active prevention and treatment strategies for HT. The aim of the present study was to investigate the potential role of p38mapk in nerve damage due to cerebral ischemia reperfusion and to explore the mechanisms underlying HT.

Genetics and Molecular Research 15 (3): gmr.15038492 


\title{
MATERIAL AND METHODS
}

\begin{abstract}
Animals
One hundred male Sprague-Dawley rats (200-240 g each) were obtained from the Laboratory Animal Centre of Medicine Inspecting Institute in Qingdao, People's Republic of China. The rats were maintained on a 12 -h light $/ 12$-h dark regimen at $19^{\circ}-25^{\circ} \mathrm{C}$ and $55-65 \%$ humidity. Food and water were provided ad libitum. Animals were acclimatized to laboratory conditions for at least 3 days before surgery. This study was carried out in strict accordance with the recommendations in the Guide for the Care and Use of Laboratory Animals of the National Institutes of Health. The animal use protocol was reviewed and approved by the Institutional Animal Care and Use Committee (IACUC) of Qingdao University.
\end{abstract}

\section{Reversible middle cerebral artery (MCA) occlusion (Longa et al., 1989)}

Animals were anesthetized by intraperitoneal injection of chloral hydrate $(10 \%)$. Body temperature was monitored and maintained at $36.5^{\circ}-37.5^{\circ} \mathrm{C}$. A standard model of the intraluminal MCA was used to create permanent MCA focal ischemia by intraluminal placement of a filament.

\section{Experimental groups}

Rats were randomly divided into three groups. Rats in the ischemia reperfusion group $(\mathrm{N}=40)$ were subjected to filament insertion for $2 \mathrm{~h}$ and were euthanized after $24 \mathrm{~h}$. Rats in the ischemia group $(\mathrm{N}=40)$ were subjected to filament insertion for $26 \mathrm{~h}$ and were immediately euthanized. Rats in the sham-operated group $(\mathrm{N}=15)$ were subjected to the same surgical procedure as the first two groups except for filament insertion, and were euthanized after $26 \mathrm{~h}$. Five rats were used as preliminary experimental animals.

\section{Analysis of neurological deficit scores}

A double-blind neurological test was administered to the experimental groups $2 \mathrm{~h}$ postischemia. A modified five-point scale system was used: 0 , normal spontaneous movements; 1, right front leg flexed but no circling clockwise; 2, circling clockwise; 3, spin clockwise longitudinally; 4 , unconsciousness and no response to noxious stimulus.

\section{Determination of infarct volume}

Twenty-six hours after transient MCAO, the rats were re-anesthetized with chloral hydrate. The fresh brains were dissected, cut into four coronal slices, and incubated in $2 \%$ 2,3,5-triphenyltetrazolium chloride (TTC) for $20 \mathrm{~min}$ at $37^{\circ} \mathrm{C}$. They were then fixed by immersion in $4 \%$ paraformaldehyde solution. The stained cerebral sections were photographed, and ipsilateral and contralateral hemispheric volumes and infarct volumes were quantified using the Image Pro-Plus 5.1 (Media Cybernetics, Inc., Bethesda, MD, USA) analysis software.

Genetics and Molecular Research 15 (3): gmr.15038492 


\section{Preparation of paraffin sections}

Twenty rats from each group were euthanized for the preparation of paraffin sections. The rats were anesthetized by intraperitoneal $10 \%$ chloral hydrate injection and their blood was cleared using physiological saline. Following whole-body perfusion/fixation, the brains were harvested and placed in 4\% paraformaldehyde for $24 \mathrm{~h}$. Paraffin embedding was performed after ethanol dehydration and exposure to xylene to clear the brain tissue. Slices $(5-\mu \mathrm{m}$ thick) were prepared and stored for later use.

\section{Histopathological examination}

Microtomed brain tissue sections were dewaxed, hydrated, and stained with hematoxylin and eosin for histopathological examination.

\section{Immunohistochemistry}

Dewaxed and hydrated microtomed brain tissue sections were blocked using $3 \% \mathrm{H}_{2} \mathrm{O}_{2}$ for 10 min and incubated with $\mathrm{p} 38$ mapk rabbit polyclonal antibody (Santa Cruz Biotechnology; 1:100 dilution in $0.01 \mathrm{M}$ phosphate-buffered saline) followed by incubation with secondary antibody (Zhong Shan Biology Technology Company, Peoples' Republic of China). Five visual fields of the ischemic region of the infarct were selected, and the immune-reactive cells were counted under a light microscope with a 400X objective lens.

\section{Statistical analysis}

All results are reported as means \pm SE. The Student $t$-test (SPSS 18.0 software) was used to compare test groups with the sham-operated control group. The level of significance was set at $\mathrm{P}<0.05$.

\section{RESULTS}

One hundred rats were included in this study, of which five were used as preliminary experimental animals. The remaining 95 were used as experimental animals, of which 10 died during the course of the experiment. Eighty-five rats underwent successful middle cerebral artery infarction. The sham-operated group comprised 15 rats, while the ischemia reperfusion and ischemia groups comprised 35 rats each. Five rats from each group were used as pathological models. In the sham-operated group, five rats were used to detect brain infarct volume and five were used to detect index expression. In the ischemia reperfusion and ischemia groups, 10 rats were used to detect brain infarct volume and 20 were used to detect index expression.

\section{Ischemia reperfusion improved neurological deficit}

Neurological deficit was examined and scored on a 5-point scale 2 and $26 \mathrm{~h}$ after surgery. Compared with the sham-operated group, the neurological deficit scores in the ischemia reperfusion and ischemia groups improved significantly $(\mathrm{P}<0.05) 26 \mathrm{~h}$ after surgery,

Genetics and Molecular Research 15 (3): gmr.15038492 
but not after $2 \mathrm{~h}$. The 26-h neurological deficit scores in the ischemia reperfusion group were higher than those in the ischemia group $(\mathrm{P}<0.05$; Figure 1).

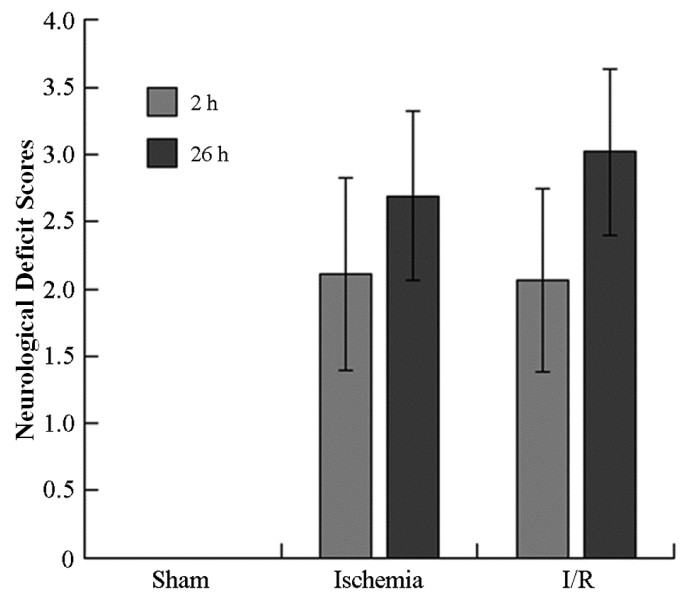

Figure 1. Effect of operation on rat neurological function scores. After ischemia, the 2-h scores in the ischemia and ischemia reperfusion $(\mathrm{I} / \mathrm{R})$ groups were significantly improved compared with the sham-operated group $(\mathrm{P}<0.05)$. However, the differences between the 2-h scores in the ischemia and I/R groups were not statistically significant (P $>0.05)$. At $26 \mathrm{~h}$, the scores in the ischemia and I/R groups were improved compared with the 2 - $\mathrm{h}$ scores $(\mathrm{P}<0.05)$. The 26-h scores in the I/R group were higher than in the ischemia group $(\mathrm{P}<0.05)$.

\section{Ischemia reperfusion improved infarct volume}

Cerebral infarction was detected by TTC staining (Figures 2 and 3). No infarction was observed in the sham-operated group. At $26 \mathrm{~h}$, the infarct volume in the ischemia reperfusion group $(42.3 \pm 1.33 \%)$ was significantly larger than in the ischemia group $(33.7 \pm 3.06 \% ; \mathrm{P}<0.05)$.

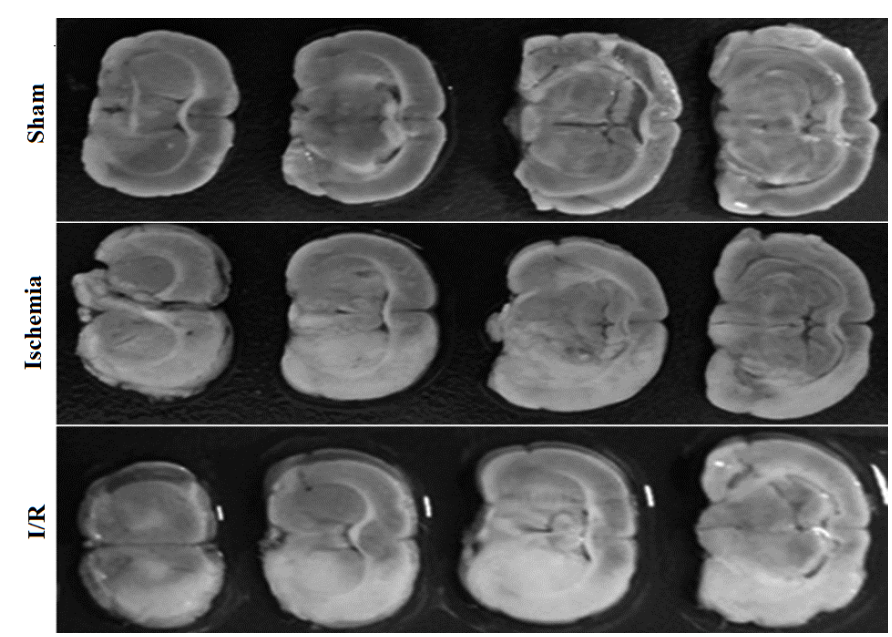

Figure 2. Photographs showing infarct volume at $26 \mathrm{~h}$ after operation. Unaffected brain regions were stained, and infarct regions were unstained. No infarction was observed in the sham-operated group. 


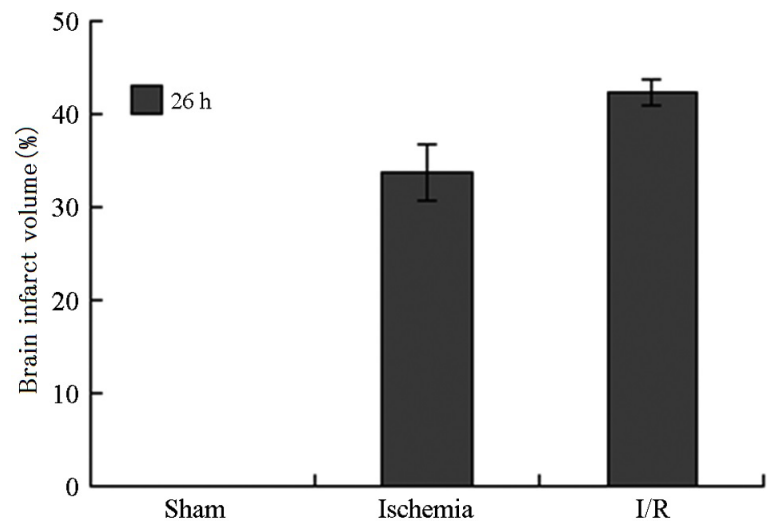

Figure 3. Infarct volume at $26 \mathrm{~h}$ after operation. The infarct volume in the ischemia reperfusion (I/R) group was significantly larger than in the ischemia group $(\mathrm{P}<0.05)$.

\section{Degree of tissue necrosis increased after reperfusion}

Necrotic tissue was not observed in the sham-operated group. In the ischemia group, necrotic tissue was observed in the zone where blood is supplied by the middle cerebral artery. The extent of damage was found to be greater in the ischemia reperfusion group than in the ischemia group (Figure 4).

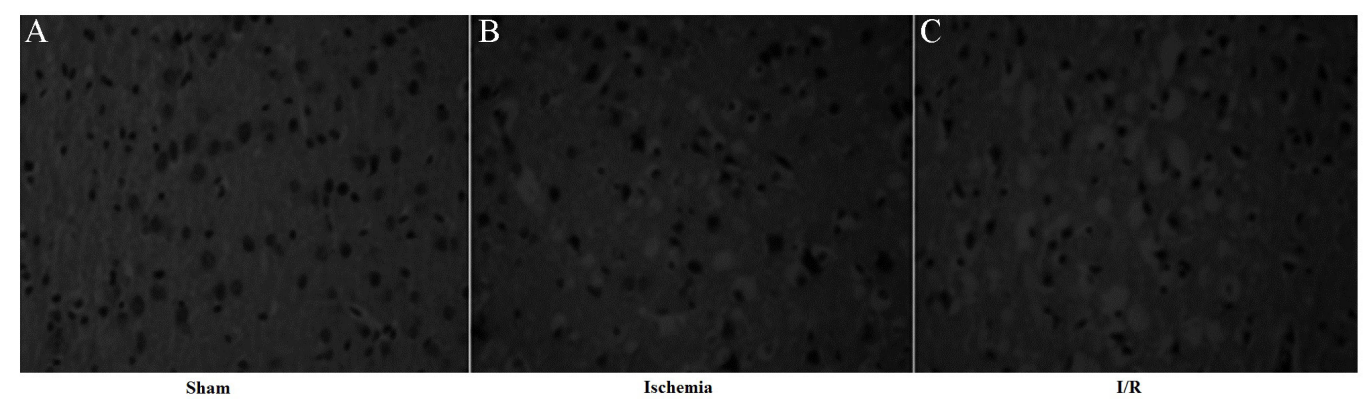

Figure 4. Histopathological examination. Damaged neurons, characterized by nuclear pyknosis and karyolysis, were evident in the ischemia and ischemia reperfusion (I/R) groups. Many vacuoles developed around the neurons. The I/R group was more seriously damaged than the ischemia group. The microphotographs were produced at a 400X magnification. A. Sham-operated group. B. Ischemia group. C. I/R group.

\section{p38mapk was significantly overexpressed after reperfusion}

Immunohistochemistry analysis revealed that few cells were reactive with primary antibodies against p38mapk in the cortices of rats in the sham-operated group. However, the number of p38mapk-positive cells increased in the cortices of rats in the ischemic group. The number of positive cells was significantly greater in the cortices of rats in the ischemic reperfusion group compared with those in the cortices of rats in the ischemic group. The differences between the three groups were statistically significant $(\mathrm{P}<0.05$; Figures 5 and 6). 


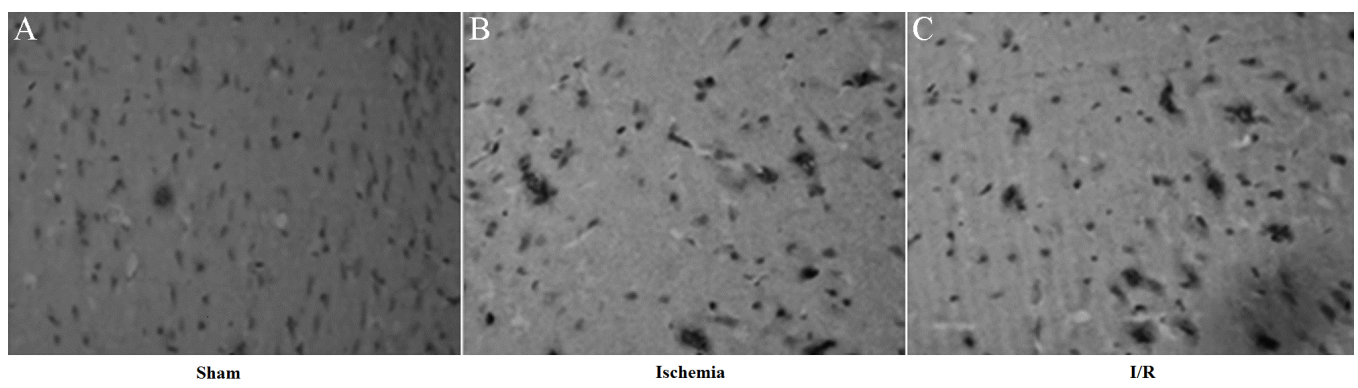

Figure 5. p38mapk expression in the cortex of rats after operation. The microphotographs were produced at a $400 \mathrm{X}$ magnification. A. Sham-operated group. B. Ischemia group. C. Ischemia/reperfusion (I/R) group.

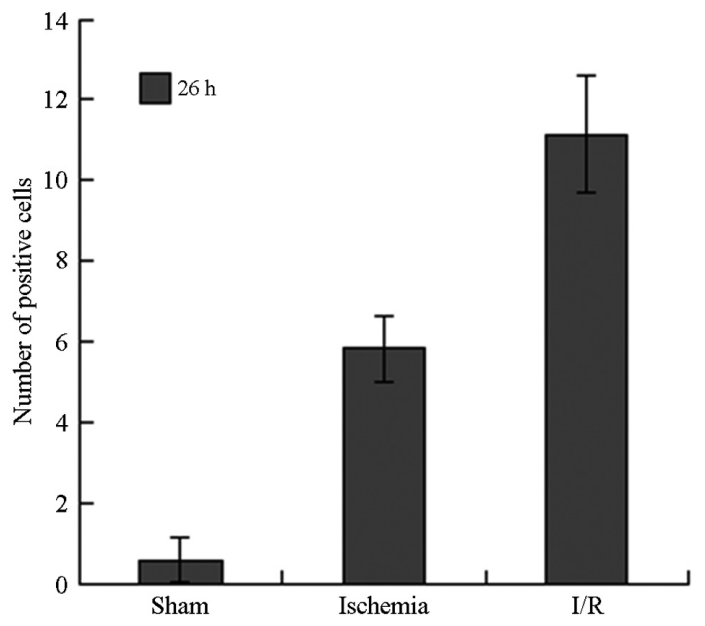

Figure 6. Number of cells expressing p38mapk in the cortex of rats after operation. The level of p38mapk expression in the ischemia reperfusion $(\mathrm{I} / \mathrm{R})$ group was significantly higher than in the other two groups $(\mathrm{P}<0.05)$.

\section{DISCUSSION}

Currently, the treatment of choice for cerebral ischemia is early reperfusion. However, HT, which is a common complication of ischemic stroke, is exacerbated by thrombolytic therapy. Increased reactive oxygen species, necrosis, and apoptosis can lead to more serious inflammation injury after reperfusion, and can result in BBB damage. No effective therapeutic interventions are available to treat HT, and there is a need to develop effective methods to prevent, predict, and treat it. To this end, the mechanism underlying cerebral hemorrhage transformation needs to be thoroughly understood. Ischemia reperfusion injury is an important factor leading to HT, and novel methods to prevent ischemia reperfusion injury have to be developed to prevent HT (Chen et al., 2014).

The cellular microenvironment, such as the presence of cell damage, and the concentrations of nutrients, growth factors, and cytokines, can be altered. Cells respond to these alterations by activating four well-characterized subfamilies of MAPKs: ERK1/2, ERK5, JNKs, and p38s. MAPKs are activated by the central three-tiered core signaling modules: MAPK kinase kinases (MKKKs), MAPK kinases (MKKs), and MAPKs (Cuenda and Rousseau, 2007).

Genetics and Molecular Research 15 (3): gmr.15038492 
Among the MAPKs, p38mapk has been implicated in the etiology of a number of diseases, and it plays an important role in cellular differentiation, cell migration, inflammation, cancer, cardiovascular dysfunction, Alzheimer's disease (AD), and Parkinson's disease (PD). The EGFR-MAPK pathway can be activated by deoxycholic acid in hepatocytes and in a number of gastrointestinal cells, including Barrett's-associated esophageal cells, gastric cancer cells, cholangiocarcinoma cells, and colon cancer cells. The EGFR-MAPK pathway plays a role in colon cancer development and metastasis (Centuori and Martinez, 2014). $\mathrm{AD}$ and PD are the most common neurodegenerative diseases. Despite intensive study, the pathogeneses of $\mathrm{AD}$ and $\mathrm{PD}$ are poorly understood. Astrocytes in the central nervous system maintain homeostasis and support neuronal function. However, senescent astrocytes can activate p38mapk and secrete inflammatory factors. Other clinical studies have found that phosphorylated p38mapk and phosphorylated JNK levels are significantly increased in AD and PD patients. Thus, p38mapk may play a role in the etiology of AD and PD (Bhat et al., 2012; Wang et al., 2014b). p38mapk activation in the cortex has been observed after MCAO, which in turn activates cytokines such as TNF- $\alpha$ and IL-1 $\beta$. It has been demonstrated that TNF- $\alpha$ and IL- $1 \beta$ destroy the structure and function of the BBB. Damage to the BBB results in cerebral hemorrhage transformation. Therefore, it has been suggested that $\mathrm{p} 38 \mathrm{mapk}$ has a potential role in attenuating the disruption of the BBB following ischemia (Dong et al., 2013; Wu et al., 2014). In mice, p38mapk contributes to the expression of LPS-induced COX2 (an inflammatory mediator). ET-1 induction can also lead to upregulation of the COX-2/ PGE2 system in brain microvascular endothelial cells by activation of ETB-dependent MAPK cascades (Lin et al., 2013; Chuang et al., 2014). Based on a clinically relevant experimental model, it has been demonstrated that activation of both JNK and p38mapk in the rostral ventrolateral medulla sustains central cardiovascular regulation during progression towards brain stem death (Chang, 2012).

The role of p38mapk has also been studied in other contexts. Choi et al. (2012) found that acupuncture can relieve pain by inhibiting reactive oxygen species-induced microglial activation and inflammatory responses. Activation of p38mapk and ERK is the main cause of inflammatory responses. Atorvastatin, which may act by downregulating p38mapk and ameliorating BBB permeability, protects the brain from the damage caused by MCAO at the early stage. Thus, p38mapk may increase BBB permeability (Cui et al., 2010). Luteolin, which may act by downregulating p38mapk and upregulating ERK expression, protects the brain from the damage caused by permanent MCAO (Qiao et al., 2012).

The BBB protects the brain from toxic substances by limiting the entry of unwanted blood components to the brain. It permits the entry of nutrients and endocrine signals to the central nervous system through active transport mechanisms and passive diffusion. The BBB secures the brain's immune-privileged status by restricting the entry of peripheral inflammatory mediators such as cytokines and antibodies. The BBB is composed of specialized endothelial cells of the cerebral microvasculature, surrounding pericytes, astrocytic endfeet, perivascular macrophages, microglia, the basement membrane, and cell-cell junctions (Keller, 2013; Najjar et al., 2013; Hsu and Kanoski, 2014; Williams et al., 2014). As mentioned above, inflammation-mediated damage leads to BBB hyperpermeability, and S100 calcium-binding protein A4 (S100A4) may play a role in the induction of BBB disruption caused by the inflammation associated with rheumatoid arthritis. S100A4 is reported to stimulate expression and proteolytic activation of matrix metalloproteinases (MMPs) in endothelial cells. MMPs are known to disrupt the BBB (Nishioku et al., 2011). Microvascular tight junctions (TJs) maintain

Genetics and Molecular Research 15 (3): gmr.15038492 
the integrity of the BBB. Hyperpermeability of the BBB in multiple sclerosis is involved in the pathology of microvascular TJs. Ischemia reperfusion injury elicits a series of events at the $\mathrm{BBB}$, which in the early phase cause BBB disruption and TJ complex degradation, whereas in the later phase they are associated with inflammatory processes and cytokine-induced BBB changes. It has been demonstrated that for most TJ proteins, such as claudin-5, occludin, and $\mathrm{ZO}-1$, a very specific fragmentation or loss of staining is noted in vessels after MCAO when they are compared with the corresponding area in sham-operated mice or the contralateral hemisphere (Kirk et al., 2003; Sladojevic et al., 2014).

The major findings of the current study are as follows. Firstly, the neurological deficit scores of the ischemia reperfusion group were higher than those of the other two groups. Secondly, the volume of infarction was larger in the ischemia reperfusion group than in the other two groups. Thirdly, histopathological examination showed that the extent of damage was greater in the ischemia reperfusion group than in the other two groups. Fourthly, p38mapk protein expression was significantly higher in the ischemia reperfusion group than in the other two groups. p38mapk may, therefore, be one of the causes of ischemia reperfusion injury and increased cerebral hemorrhage transformation.

A number of reports have studied the role of $\mathrm{p} 38$ mapk in cerebral infarction. Most studies have concluded that $\mathrm{p} 38$ mapk plays an adverse role in cerebral tissue, including the BBB. However, Yang et al. (2012) have proposed that microglial cells may play a beneficial role in the central nervous system, and astrocyte-conditioned, medium-induced, brain-derived neurotrophic factors may be mediated through the p38-MAPK signaling pathway. MMPs are known to cause BBB breakdown (Shin et al., 2007). Delayed neuroprotection induced by sevoflurane may be via opening of mitochondrial ATP-sensitive potassium channels and p38mapk phosphorylation, which indicates that $\mathrm{p} 38 \mathrm{mapk}$ is a protective factor in neurons that have undergone ischemic stimulation (Ye et al., 2012).

However, other studies do not support the conclusions drawn from the three studies mentioned above. Osteopontin is one of the cytokines involved in vascular inflammation, and its expression is induced by aldosterone. p38mapk is an intermediary between osteopontin and aldosterone, and is an inflammatory mediator that can destroy normal tissue (Fu et al., 2012). Kwon et al. (2013) have demonstrated that aluminum nanoparticles have a neurotoxic effect on rat brains that is mediated by activation of the p38mapk pathway. Cellphone radiation exposure can lead to biological interaction, and $3 \mathrm{G}$ mobile phone exposure causes a transient increase in phosphorylation of p38mapk. This in turn leads to mitochondrial dysfunctionmediated cytochrome c release and subsequent activation of caspases, which are involved in the process of radiation-induced apoptotic cell death (Kesari et al., 2014).

To conclude, the present study provides evidence that p38mapk is a damage-inducing factor. A study could be designed using a p38mapk inhibitor to strengthen the conclusions drawn from the present study. However, it is difficult to establish cellular targets of signaling pathways associated with cerebral hemorrhage transformation damage. A clearer insight into the physiological and pathophysiological roles of p38mapk may lead to a better understanding of cerebral hemorrhage transformation, which in turn may pave the way for the development of novel approaches for preventing and treating neurological pathologies.

\section{Conflicts of interest}

The authors declare no conflict of interest.

Genetics and Molecular Research 15 (3): gmr.15038492 


\section{REFERENCES}

Bhat R, Crowe EP, Bitto A, Moh M, et al. (2012). Astrocyte senescence as a component of Alzheimer's disease. PLoS One 7: e45069. http://dx.doi.org/10.1371/journal.pone.0045069

Centuori SM and Martinez JD (2014). Differential regulation of EGFR-MAPK signaling by deoxycholic acid (DCA) and ursodeoxycholic acid (UDCA) in colon cancer. Dig. Dis. Sci. 59: 2367-2380.http://dx.doi.org/10.1007/s10620-014-3190-7

Chang AY (2012). Pro-life role for c-Jun N-terminal kinase and p38 mitogen-activated protein kinase at rostral ventrolateral medulla in experimental brain stem death. J. Biomed. Sci. 19: 96. http://dx.doi.org/10.1186/1423-0127-19-96

Chen HM, Huang HS, Ruan L, He YB, et al. (2014). Ulinastatin attenuates cerebral ischemia-reperfusion injury in rats. Int. J. Clin. Exp. Med. 7: 1483-1489.

Choi DC, Lee JY, Lim EJ, Baik HH, et al. (2012). Inhibition of ROS-induced p38MAPK and ERK activation in microglia by acupuncture relieves neuropathic pain after spinal cord injury in rats. Exp. Neurol. 236: 268-282. http://dx.doi. org/10.1016/j.expneurol.2012.05.014

Choi YK, Cho GS, Hwang S, Kim BW, et al. (2010). Methyleugenol reduces cerebral ischemic injury by suppression of oxidative injury and inflammation. Free Radic. Res. 44: 925-935.http://dx.doi.org/10.3109/10715762.2010.490837

Chuang YF, Yang HY, Ko TL, Hsu YF, et al. (2014). Valproic acid suppresses lipopolysaccharide-induced cyclooxygenase-2 expression via MKP-1 in murine brain microvascular endothelial cells. Biochem. Pharmacol. 88: 372-383. http:// dx.doi.org/10.1016/j.bep.2014.02.004

Cuenda A and Rousseau S (2007). p38 MAP-kinases pathway regulation, function and role in human diseases. Biochim. Biophys. Acta 1773: 1358-1375.http://dx.doi.org/10.1016/j.bbamcr.2007.03.010

Cui L, Zhang X, Yang R, Wang L, et al. (2010). Neuroprotection of early and short-time applying atorvastatin in the acute phase of cerebral ischemia: down-regulated 12/15-LOX, p38MAPK and cPLA2 expression, ameliorated BBB permeability. Brain Res. 1325: 164-173.http://dx.doi.org/10.1016/j.brainres.2010.02.036

Dong L, Qiao H, Zhang X, Zhang X, et al. (2013). Parthenolide is neuroprotective in rat experimental stroke model: downregulating NF-kB, phospho-p38MAPK, and caspase-1 and ameliorating BBB permeability. Mediators Inflamm. 2013: 370804.http://dx.doi.org/10.1155/2013/370804

Exil V, Ping L, Yu Y, Chakraborty S, et al. (2014). Activation of MAPK and FoxO by manganese (Mn) in rat neonatal primary astrocyte cultures. PLoS One 9: e94753.http://dx.doi.org/10.1371/journal.pone.0094753

Fu GX, Xu CC, Zhong Y, Zhu DL, et al. (2012). Aldosterone-induced osteopontin expression in vascular smooth muscle cells involves MR, ERK, and p38 MAPK. Endocrine 42: 676-683. http://dx.doi.org/10.1007/s12020-012-9675-2

Hsu TM and Kanoski SE (2014). Blood-brain barrier disruption: mechanistic links between western diet consumption and dementia. Front. Aging Neurosci. 6: 88. http://dx.doi.org/10.3389/fnagi.2014.00088

Keller A (2013). Breaking and building the wall: the biology of the blood-brain barrier in health and disease. Swiss Med. Wkly. 143: w13892.

Kesari KK, Meena R, Nirala J, Kumar J, et al. (2014). Effect of 3G cell phone exposure with computer controlled 2-D stepper motor on non-thermal activation of the hsp27/p38MAPK stress pathway in rat brain. Cell. Biochem. Biophys. 68: 347-358. http://dx.doi.org/10.1007/s12013-013-9715-4

Kirk J, Plumb J, Mirakhur M and McQuaid S (2003). Tight junctional abnormality in multiple sclerosis white matter affects all calibres of vessel and is associated with blood-brain barrier leakage and active demyelination. J. Pathol. 201: 319-327.http://dx.doi.org/10.1002/path.1434

Kwon JT, Seo GB, Jo, Lee M, et al. (2013). Aluminum nanoparticles induce ERK and p38MAPK activation in rat brain. Toxicol. Res. 29: 181-185. http://dx.doi.org/10.5487/TR.2013.29.3.181

Lin CC, Hsieh HL, Shih RH, Chi PL, et al. (2013). Up-regulation of COX-2/PGE2 by endothelin-1 via MAPK-dependent NF-kB pathway in mouse brain microvascular endothelial cells. Cell. Commun. Signal. 11: 8. http://dx.doi. org/10.1186/1478-811X-11-8

Longa EZ, Weinstein PR, Carlson S and Cummins R (1989). Reversible middle cerebral artery occlusion without craniectomy in rats. Stroke 20: 84-91.http://dx.doi.org/10.1161/01.STR.20.1.84

Nadeem A, Siddiqui N, Alharbi NO, Alharbi MM, et al. (2014). Acute glutathione depletion leads to enhancement of airway reactivity and inflammation via p38MAPK-iNOS pathway in allergic mice. Int. Immunopharmacol. 22: 222229. http://dx.doi.org/10.1016/j.intimp.2014.06.030

Najjar S, Pearlman DM, Devinsky O, Najjar A, et al. (2013). Neurovascular unit dysfunction with blood-brain barrier hyperpermeability contributes to major depressive disorder: a review of clinical and experimental evidence. $J$. Neuroinflammation 10: 142. http://dx.doi.org/10.1186/1742-2094-10-142

Nishioku T, Furusho K, Tomita A, Ohishi H, et al. (2011). Potential role for S100A4 in the disruption of the blood-brain barrier in collagen-induced arthritic mice, an animal model of rheumatoid arthritis. Neuroscience 189: 286-292. http://dx.doi.org/10.1016/j.neuroscience.2011.05.044

Genetics and Molecular Research 15 (3): gmr.15038492 
Qiao H, Zhang X, Zhu C, Dong L, et al. (2012). Luteolin downregulates TLR4, TLR5, NF-kB and p-p38MAPK expression, upregulates the p-ERK expression, and protects rat brains against focal ischemia. Brain Res. 1448: 71-81. http:// dx.doi.org/10.1016/j.brainres.2012.02.003

Robinson AA, Ikuta K and Soverow J (2014). Anticoagulation for the acute management of ischemic stroke. Yale J. Biol. Med. 87: 199-206.

Shin CY, Lee WJ, Choi JW, Choi MS, et al. (2007). Role of 38 MAPK on the down-regulation of matrix metalloproteinase-9 expression in rat astrocytes. Arch. Pharm. Res. 30: 624-633. http://dx.doi.org/10.1007/BF02977658

Sladojevic N, Stamatovic SM, Keep RF, Grailer JJ, et al. (2014). Inhibition of junctional adhesion molecule-A/LFA interaction attenuates leukocyte trafficking and inflammation in brain ischemia/reperfusion injury. Neurobiol. Dis. 67: 57-70. http://dx.doi.org/10.1016/j.nbd.2014.03.010

Wang L, Li Z, Zhang X, Wang S, et al. (2014a). Protective effect of shikonin in experimental ischemic stroke: attenuated TLR4, p-p38MAPK, NF-kB, TNF-a and MMP-9 expression, up-regulated claudin-5 expression, ameliorated BBB permeability. Neurochem. Res. 39: 97-106. http://dx.doi.org/10.1007/s11064-013-1194-x

Wang S, Zhang C, Sheng X, Zhang X, et al. (2014b). Peripheral expression of MAPK pathways in Alzheimer's and Parkinson's diseases. J. Clin. Neurosci. 21: 810-814.http://dx.doi.org/10.1016/j.jocn.2013.08.017

Wang Y, Ge P and Zhu Y (2013). TLR2 and TLR4 in the brain injury caused by cerebral ischemia and reperfusion. Mediators Inflamm. 2013: 124614.http://dx.doi.org/10.1155/2013/124614

Williams JL, Holman DW and Klein RS (2014). Chemokines in the balance: maintenance of homeostasis and protection at CNS barriers. Front. Cell Neurosci. 8: 154. http://dx.doi.org/10.3389/fncel.2014.00154

Wu XL, Wang P, Liu YH and Xue YX (2014). Effects of poly (ADP-ribose) polymerase inhibitor 3-aminobenzamide on blood-brain barrier and dopaminergic neurons of rats with lipopolysaccharide-induced Parkinson's disease. J. Mol. Neurosci. 53: 1-9. http://dx.doi.org/10.1007/s12031-013-0175-5

Yang H, Feng GD, Liang Z, Vitale A, et al. (2012). In vitro beneficial activation of microglial cells by mechanically-injured astrocytes enhances the synthesis and secretion of BDNF through p38MAPK. Neurochem. Int. 61: 175-186. http:// dx.doi.org/10.1016/j.neuint.2012.04.020

Ye Z, Guo Q, Wang N, Xia P, et al. (2012). Delayed neuroprotection induced by sevoflurane via opening mitochondrial ATP-sensitive potassium channels and p38 MAPK phosphorylation. Neurol. Sci. 33: 239-249. http://dx.doi. org $/ 10.1007 / \mathrm{s} 10072-011-0665-6$

Zhao Z, Yin JQ, Wu MS, Song G, et al. (2014). Dihydromyricetin activates AMP-activated protein kinase and P38(MAPK) exerting antitumor potential in osteosarcoma. Cancer Prev. Res. 7: 927-938. http://dx.doi.org/10.1158/1940-6207. CAPR-14-0067

Genetics and Molecular Research 15 (3): gmr.15038492 